\title{
Immediate Change of Radiation Doses from Hydrothermal Deposits
}

\author{
Shin Toyoda, Fumihiro Sato, Ai Uchida, and Jun-ichiro Ishibashi
}

\begin{abstract}
The radiation from the sulfide deposits taken at Okinawa Trough was measured on-board by a NaI gamma ray spectrometer immediately after the retrieval of the sample onto the research vessel. Gamma ray peaks were identified as those from daughter nuclei of ${ }^{226} \mathrm{Ra}$ and ${ }^{228} \mathrm{Ra}$. The slight change with time of the radiation dose from those deposits is probably due to the geometric arrangement in measurements. As results, no change in radiation dose was observed in the time range up to $90 \mathrm{~h}$ after retrieval of the samples from the submarine vehicle.
\end{abstract}

Keywords

Dating $\bullet$ On-board measurement $\bullet$ Radiation $\bullet$ Sulfide

\subsection{Introduction}

Intense natural radiation is often observed in the sea-floor hydrothermal area due to radium and radon, which are originally produced by decay of uranium and thorium and are dissolved into the hydrothermal fluids. Measurements in the laboratory on land detect only long lived radioactive nuclei, whose half lives are in the order of years or larger, while immediate decrease of radioactivity of hydrothermal sulfide deposits is sometimes observed on the research vessel (Ishibashi, personal communications). The present study aimed to detect possible natural radioactive nuclei of short life time by on-board measurements with a NaI gamma ray spectrometer immediately after retrieving the sulfide deposit samples.

The online version of this chapter (doi:10.1007/978-4-431-54865-2_49) contains supplementary material, which is available to authorized users.

S. Toyoda $(\bowtie) \cdot$ F. Sato $\bullet$ A. Uchida

Okayama University of Science, Okayama, Japan

e-mail: toyoda@dap.ous.ac.jp

J.-i. Ishibashi

Kyushu University, Fukuoka, Japan

\subsection{Samples and Experimental Procedures}

Sulfide deposit samples were taken during NT11-20 and NT12-06 cruises with research vessel Natsushima operated by JAMSTEC in September, 2011 and in March, 2012, respectively. Four samples were examined as listed in Table 49.1, which were taken at sea-floor hydrothermal sites shown in Suppl. 49.1, and are shown in Fig. 49.1.

As soon as a sample was retrieved, the sample block of about $15 \mathrm{~cm}$ in length was put into a plastic bag. It was then placed in front of the detector of a $\mathrm{NaI}$ gamma ray spectrometer having a $3^{\prime \prime}$ detector, Aloka JSM-102, where the full scale of 240 channels are set up to $3,600 \mathrm{keV}$. The measurements with duration of $30 \mathrm{~min}$ were repeated several times successively or with some interval for each sample up to $90 \mathrm{~h}$. After the cruises, the spectrum data were transferred to a PC and were converted to text files. The threshold method (Mercier and Falgueres 2007) was used to obtain the dose rates given by the samples. The counts from the 25th channel to 240th channel were integrated and compared with those obtained for standard calibration concrete blocks (Uchida et al. 2014) in order to obtain the dose rates. 
Table 49.1 Samples examined in the present study

\begin{tabular}{lllll}
\hline Sample & Site & Position & Description & Dimension $(\mathrm{cm})$ \\
\hline HPD\#1328G04 & Minami Ensei Knoll & $28^{\circ} 23.462^{\prime} \mathrm{N}, 127^{\circ} 38.398^{\prime} \mathrm{E}$ & Sulfide chimney & $18 \times 18 \times 13$ \\
\hline HPD\#1329G05 & Izena (Hakurei) & $27^{\circ} 14.815^{\prime} \mathrm{N}, 127^{\circ} 53.736^{\prime} \mathrm{E}$ & Sulfide chimney & $17 \times 13 \times 8$ \\
\hline HPD\#1331G05 & Hatoma Knoll & $24^{\circ} 51.507^{\prime} \mathrm{N}, 123^{\circ} 50.546^{\prime} \mathrm{E}$ & Kuroko ore & $14 \times 11 \times 10$ \\
\hline HPD\#1358R02a & Iheya-North & $27^{\circ} 47.438^{\prime} \mathrm{N}, 126^{\circ} 53.736^{\prime} \mathrm{E}$ & Sulfide & $20 \times 10 \times 6$ \\
\hline
\end{tabular}

a

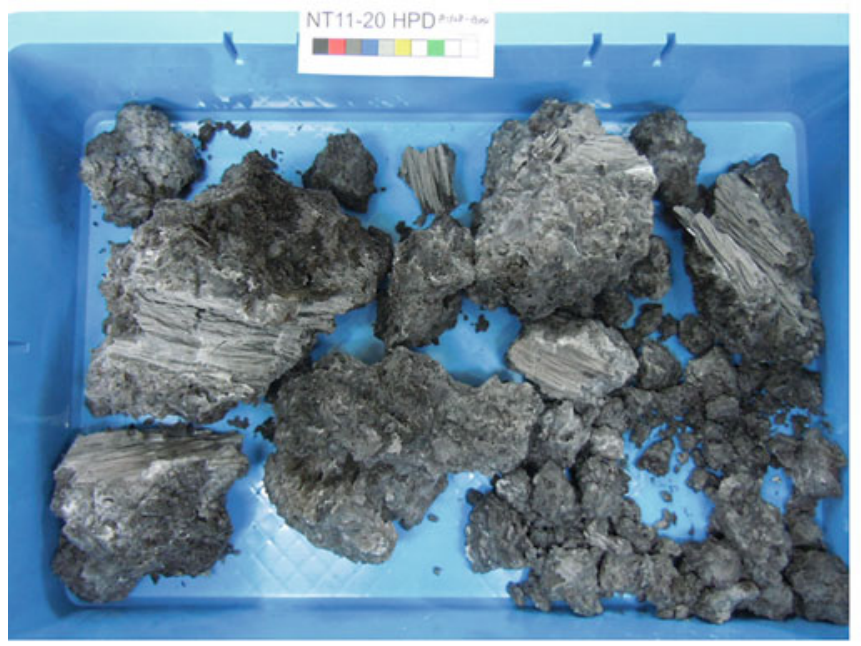

c

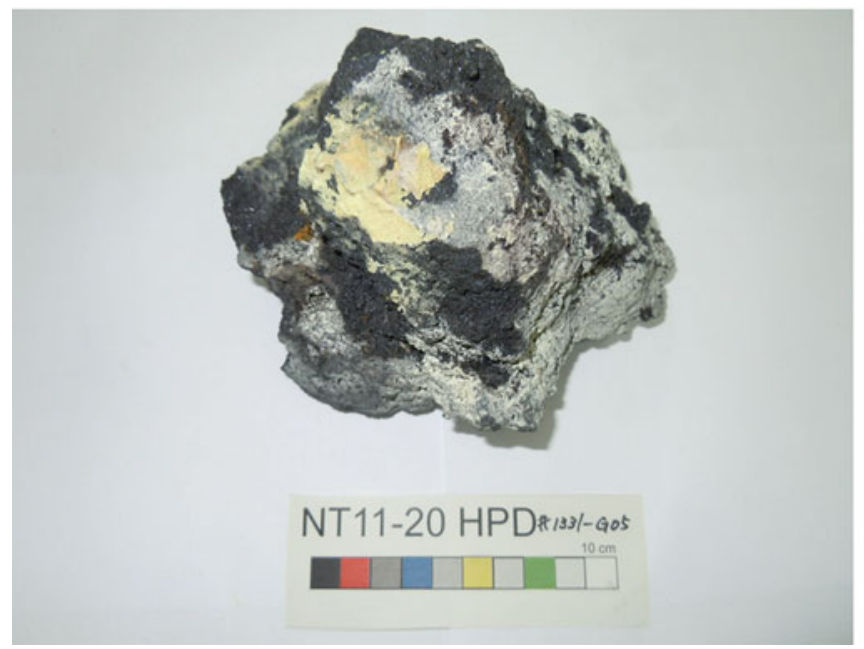

Fig. 49.1 The samples examined in the present study

\subsection{Results and Discussions}

The spectra observed in the first measurements of the samples are shown in Fig. 49.2. The peaks are identified as those due to ${ }^{214} \mathrm{Bi},{ }^{228} \mathrm{Ac},{ }^{40} \mathrm{~K}$, and ${ }^{208} \mathrm{Tl}$. The spectra observed in the following successive measurements were essentially identical to those in the first measurements. ${ }^{214} \mathrm{Bi}$ is a nucleus of uranium series while ${ }^{228} \mathrm{Ac}$ and ${ }^{208} \mathrm{Tl}$ of thorium series. These are most probably produced by b

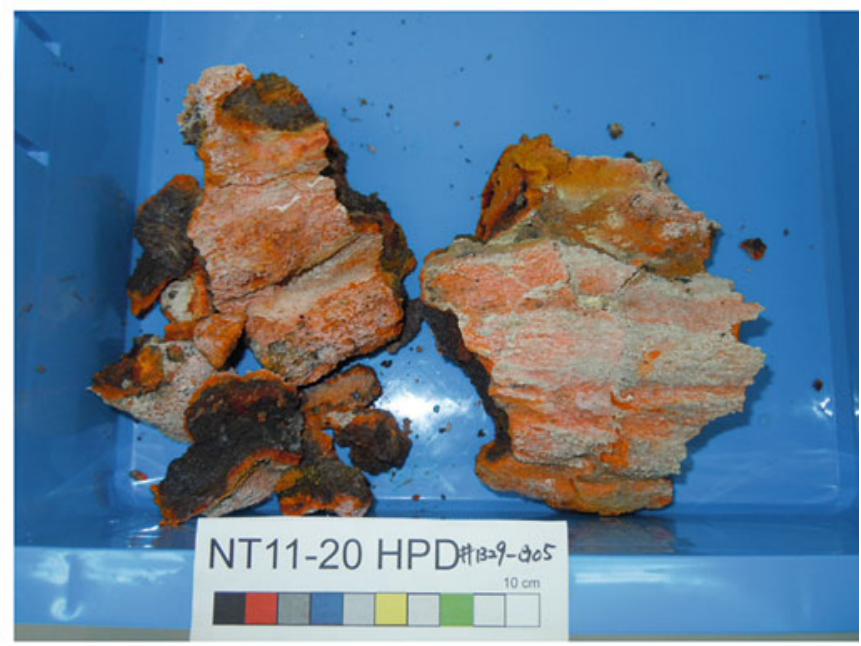

d

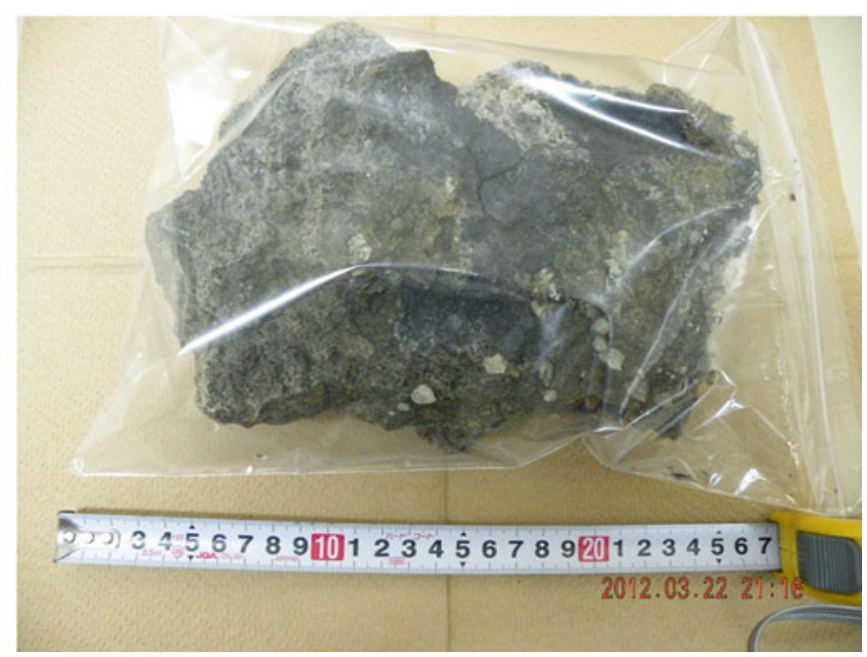

decays of ${ }^{226} \mathrm{Ra}$ and ${ }^{228} \mathrm{Ra}$ contained in the sulfide deposits rather than those of ${ }^{238} \mathrm{U}$ and ${ }^{232} \mathrm{Th}$ as those concentrations in sulfide minerals are in ppm order while those in barite are in ppb order (Toyoda et al., 2014).

The daughter nuclei of ${ }^{226} \mathrm{Ra}$ and ${ }^{228} \mathrm{Ra}$ were observed in HPD\#1328G04, HPD\#1329G05, and HPD\#1331G05, but no daughter nuclei of ${ }^{228} \mathrm{Ra}$ in HPD\#1358R02a. This makes the most remarkable difference in the spectra shown in Fig. 49.2. As the half life of ${ }^{228} \mathrm{Ra}$ is 5.75 years, the ages of the former three samples are estimated to be younger than 
Fig. 49.2 Gamma ray spectra of the samples measured just after the retrieval on the ship by an $\mathrm{NaI}$ gamma ray spectrometer
Fig. 49.3 The ratio of the peak of $2,614 \mathrm{keV}\left({ }^{208} \mathrm{Tl}\right)$ to $610 \mathrm{keV}$ $\left({ }^{214} \mathrm{Bi}\right)$
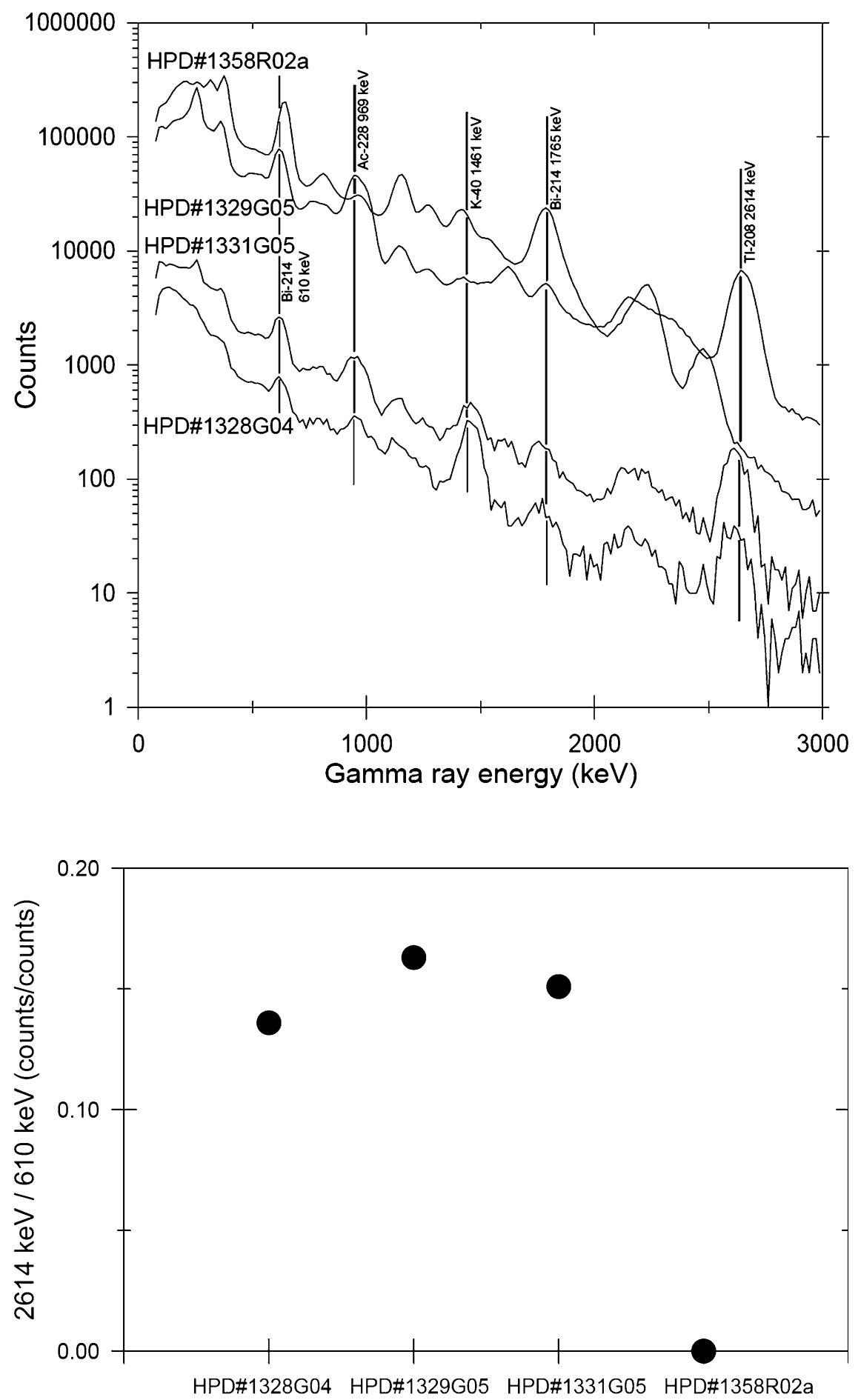

several 10 years while the age of the last sample is to be more than several 10 years. The peak due to ${ }^{40} \mathrm{~K}$ is observed in HPD\#1328G04 and in HPD\#1331G05, but not seen in HPD\#1329G05. As for the spectrum of HPD\#1358R02a, it is not clear whether the peak around $1,461 \mathrm{keV}$ is due to ${ }^{40} \mathrm{~K}$ or not. The latter two samples have much higher radioactivity due to daughter nuclei of ${ }^{226} \mathrm{Ra}$ and/or ${ }^{228} \mathrm{Ra}$ relative to that due to ${ }^{40} \mathrm{~K}$ than the former two samples probably because of the mineralogy which has not yet been analyzed. The Compton background of those and those daughter nuclei mask the peak of ${ }^{40} \mathrm{~K}$ in the former two samples. Except for ${ }^{40} \mathrm{~K}$, the shapes of the gamma ray spectra of HPD\#1328G04, HPD\#1329G05, and HPD\#1331G05 are very similar. The ratio of ${ }^{214} \mathrm{Bi}$ at $610 \mathrm{keV}$ to ${ }^{208} \mathrm{Tl}$ at $2,614 \mathrm{keV}$ is shown in Fig. 49.3, indicating that the relative ratio of ${ }^{226} \mathrm{Ra}$ and ${ }^{228} \mathrm{Ra}$ is the same for these three samples. 
Fig. 49.4 The change in the dose rates from the retrieval of the samples up to $90 \mathrm{~h}$. No change with time was observed

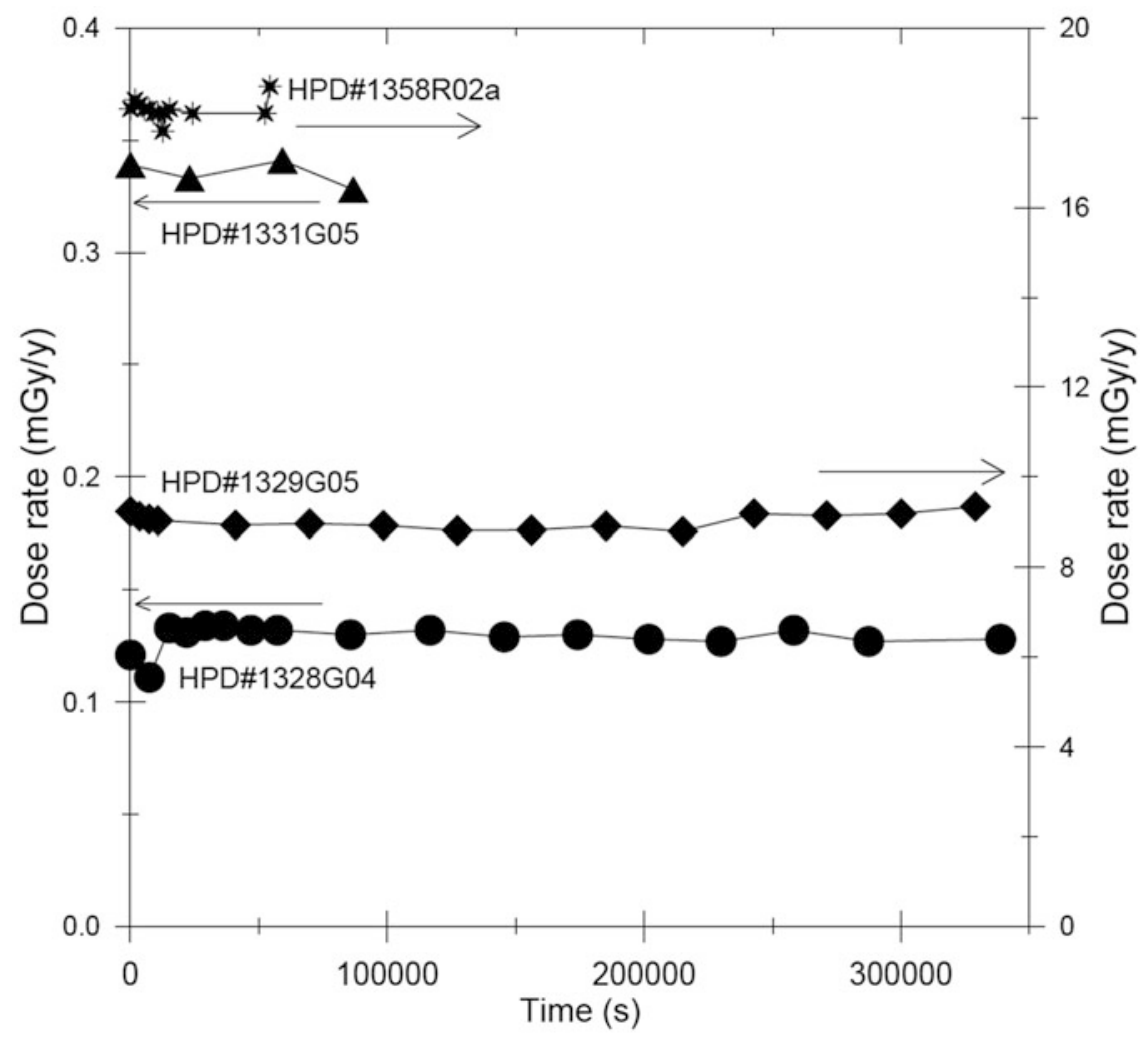

The dose rate given by the samples was calculated from the spectrum by the threshold technique (Mercier and Falgueres 2007) based on the measurements for U, Th, K calibration blocks with known amount of these elements with radioactive equilibrium. Although, therefore, the calculated dose rates should be slightly overestimated due to lack of the consideration for the nuclei between ${ }^{238} \mathrm{U}$ and ${ }^{226} \mathrm{Ra}$, and ${ }^{232} \mathrm{Th}$ and ${ }^{228} \mathrm{Ra}$ in the measured samples, relative comparison would be meaningful.

The gamma ray spectra obtained by the repeated measurements are almost identical for all present four samples. This leads to the results that the calculated dose rates given by these samples were constant with time as shown in Fig. 49.4. The slight changes with time are shown for HPD\#1328R04 and in HPD\#1329R05. However, these are probably due to the geometric arrangement in measurement because corresponding similar changes of the intensity of ${ }^{40} \mathrm{~K}$ are observed in these samples.

The immediate decrease of radioactivity of the bulk sample, which is sometimes observed on the ship in a time scale of several hours, would possibly be due to the decay of short lived nuclei or loss of radon absorbed on the surface of the sample. However, the reason was not found in the present study.

The present study showed the ability of NaI detector, the resolution of which is much less than the pure Ge gamma ray spectrometer, to detect ${ }^{228} \mathrm{Ra}$ in the samples. As ${ }^{228} \mathrm{Ra}$ in the samples indicates that their ages are young, less than several 10 years, mapping of rough ages of sulfide deposits at the sea floor would be possible when such detection is done during the dives by submarine vehicles.

Acknowledgements The work was supported by Taiga project, Grantin-Aid for Scientific Research on innovative Areas (20109004) funded by the Ministry of Education, Culture, Sports, Science and Technology (MEXT).

Open Access This chapter is distributed under the terms of the Creative Commons Attribution Noncommercial License, which permits any noncommercial use, distribution, and reproduction in any medium, provided the original author(s) and source are credited.

\section{References}

Mercier N, Falgueres C (2007) Field gamma dose-rate measurement with a $\mathrm{NaI}(\mathrm{Tl})$ detector: re-evaluation of the "threshold" technique. Ancient TL 25:1-4

Toyoda S, Fujiwara T, Uchida A, Ishibashi J, Nakai S, Takamasa A (2014) ESR dating of barite in sulfide deposits formed by the sea floor hydrothermal activities. Radiat Prot Dosimetry 159:203-211

Uchida A, Toyoda S, Tissoux H, Falguères C, Ninagawa K, Miallier D (2014) Dose rate estimation by Aloka NaI scintilation gamma ray spectrometer using the Threshold Technique. Advances in ESR Applications 30:12-15 (in Japanese with English Abstract) 